\title{
A monoclonal anti-interleukin 8 antibody (WS-4) inhibits cytokine response and acute lung injury in experimental severe acute necrotising pancreatitis in rabbits
}

M O Osman, J U Kristensen, N O Jacobsen, S B Lausten, B Deleuran, M Deleuran, B Gesser, K Matsushima, C G Larsen, S L Jensen

\begin{abstract}
Background-Interleukin 8 (IL-8) has recently been proposed to have an important role in mediating the development of the systemic sequelae associated with severe acute pancreatitis.

Aims-To define the role of IL-8 in acute pancreatitis by neutralising its effects with a monoclonal anti-IL-8 antibody (WS-4), in a rabbit model of severe acute pancreatitis.

Methods-Acute pancreatitis was induced by retrograde injection of $5 \%$ chenodeoxycholic acid into the pancreatic duct and duct ligation. Twenty rabbits were divided equally into two groups: acute pancreatitis controls received physiological saline and the treated group received WS-4, 30 minutes before induction of acute pancreatitis.

Results-Pretreatment of animals with WS-4 resulted in significant down regulation of serum IL-8 and tumour necrosis factor $\alpha(\mathrm{TNF}-\alpha)$ from three to six hours after induction of acute pancreatitis $(p=0.011$ and 0.047 for IL-8 and 0.033 and 0.022 for TNF- $\alpha$, respectively). In addition, a significant reduction in the CD11b and CD18 positive cells and the amount of interstitial neutrophil infiltration in the lungs from WS-4 treated animals was seen. In contrast, WS- 4 did not alter the amount of pancreatic necrosis and the serum concentrations of amylase, lipase, calcium, and glucose.

Conclusion-WS-4 cannot change the amount of pancreatic necrosis induced by injection of $5 \%$ bile acid, but does reduce the acute lung injury, presumably through inhibition of circulating IL-8 and TNF- $\alpha$, and CD11b/CD18 in lung tissue. Therefore, a role of IL-8 in the progression of acute pancreatitis and the development of its systemic complications is suggested. (Gut 1998;43:232-239)
\end{abstract}

Keywords: acute pancreatitis; rabbits; cytokines; anti-interleukin- 8 antibody; acute lung injury

Acute pancreatitis is a common and potentially fatal disease; its pathogenesis is incompletely resolved. ${ }^{1}$ The overall mortality from acute pancreatitis varies between $6 \%$ and $23 \%$, increasing to $20 \%$ and $50 \%$ in the most severe necrotising form of the disease. ${ }^{2}$ The cause of death in most of these patients is often due to multiorgan failure which frequently complicates severe necrotising acute pancreatitis. ${ }^{2}$

Recently, activated leucocytes and proinflammatory cytokines-for example, interleukin 1 (IL-1) and tumour necrosis factor $\alpha$ $(\mathrm{TNF}-\alpha)$ - have been suggested to play a predominant role in the progression of acute pancreatitis and the development of its systemic complications. ${ }^{3-5}$ Interleukin 8 has been characterised as a potent $\mathrm{T}$ cell and neutrophil chemotactic and activating cytokine, produced mainly by the mononuclear cells. ${ }^{67} \mathrm{~A}$ pathophysiological role of IL-8 in different inflammatory disorders has been suggested..$^{8-10}$ Furthermore, serum IL-8 has been shown to be elevated in the course of acute pancreatitis in several clinical and a few experimental studies. ${ }^{11-13}$ Measurable blood concentrations of IL-8 showed a positive correlation with the clinical course of acute pancreatitis, suggesting a role for IL-8 in development of the systemic complications of severe acute pancreatitis. ${ }^{145}$

More recently, a monoclonal antihuman IL-8 antibody (WS-4) has been shown to inhibit the binding to rabbit neutrophils and neutrophil chemotactic activity of recombinant rabbit IL-8 at a WS-4:IL-8 molar ratio of nearly $1: 1$, without any cross reactivity against any of the well known human chemokines, including platelet factor $4 .^{16}$ Subsequently, WS-4 has been reported to block completely a $\mathrm{T}$ cell mediated immune reaction of rabbit skin, ${ }^{17}$ and to prevent lung reperfusion injury in rabbits. $^{18}$

The aim of this study was to define the role of IL-8 in the pathogenesis of severe acute pancreatitis and its systemic complications, in a rabbit model of bile acid induced acute pancreatitis, by blocking its effects with the neutralising monoclonal anti-IL-8 antibody (WS-4).

\section{Materials and methods}

ANIMALS

Adult male and female New Zealand White rabbits, weighing $3.9-5.2 \mathrm{~kg}$, were used; they were housed at the animal farm of Aarhus University for at least one week prior to use. The animals were cared for in accordance with the requirements for animal care as stipulated by the Danish Experimental Animal Committee, which also approved the experimental protocol. 
ANAESTHESIA AND SURGICAL PROCEDURES

Animals, which had been fasted overnight, but with free access to tap water, were anaesthetised with a mixture of Dormicum $(2 \mathrm{mg} / \mathrm{kg}$ ) and Hypnorm $(0.3 \mathrm{ml} / \mathrm{kg})$, both injected intramuscularly. The animals were kept anaesthetised throughout the experimental period of 12 hours by repeated intramuscular injections of Hypnorm $(0.15 \mathrm{ml} / \mathrm{kg})$. The surgical procedures have been described in detail elsewhere. ${ }^{19}$ Briefly, all rabbits underwent a $4 \mathrm{~cm}$ long midline laparotomy; the pancreatic duct was visualised entering the duodenum at a point approximately $20 \mathrm{~cm}$ from the pylorus. A 24 gauge polyethylene catheter was inserted easily through a small cut in the intramural aspect of the pancreatic duct. Acute pancreatitis was induced by retrograde intraductal injection of $2.0 \mathrm{ml}$ of $5 \%$ chenodeoxycholic bile acid (clear faint yellow solution at $200 \mathrm{mg}$ plus $4.0 \mathrm{ml}$ of ethanol, Sigma Chemical Co., St Louis, Missouri, USA, product no C9377) for 2.5 minutes, in a pressure, volume, and time controlled manner. After injection, the catheter was removed, the pancreatic duct was ligated, and the abdomen was closed in two layers.

EXPERIMENTAL DESIGN

Twenty rabbits were divided into two groups. Group A (controls; $n=10$ ) received a retrograde intraductal injection of $2.0 \mathrm{ml} 5 \%$ chenodeoxycholic bile acid, followed by duct ligation. They were then given a single intravenous saline bolus injection followed by infusion of $40 \mathrm{ml}$ saline for 30 minutes until induction of acute pancreatitis. Group B $(\mathrm{n}=10)$ received a single intravenous bolus injection $(1 \mathrm{mg} / \mathrm{kg}$ dissolved in equal volume of saline) of the monoclonal neutralising antiIL-8 antibody (WS-4), 30 minutes before induction of acute pancreatitis, followed by infusion of $40 \mathrm{ml}$ of saline for 30 minutes until induction of acute pancreatitis.

Arterial blood samples were drawn 30 minutes before, and five minutes and three, six, nine, and 12 hours after induction of acute pancreatitis for determination of leucocyte count, and serum amylase, lipase, glucose, calcium, and the cytokines IL- 8 and TNF- $\alpha$. The volume of each blood sample $(6.0 \mathrm{ml})$ was replaced by $0.9 \%$ saline. Physiological saline was also infused into all animals to $4 \mathrm{ml} / \mathrm{h}$ throughout the experimental period. All blood samples, except for those used for leucocyte counts, were immediately centrifuged at 3000 $\mathrm{rpm}$ for 10 minutes at $5^{\circ} \mathrm{C}$. The supernatants were separated using sterile pipettes. All samples were coded and stored at $-80^{\circ} \mathrm{C}$ until analysed in a blinded fashion.

At the end of the experiment, surviving animals were sacrificed, and the accumulated ascitic fluid was quantified and aliquots separated for measurement of amylase and lipase. Finally, all animals underwent necropsy; tissue specimens of pancreas, lung, and kidney were obtained and each was divided into two parts. One part was fixed in $10 \%$ formalin for histological examination; the other was frozen immediately in liquid nitrogen for immunohis- tochemical analysis of the adhesion molecules CD11b and CD18.

LEUCOCYTE COUNT AND SERUM AMYLASE, LIPASE, GLUCOSE, AND CALCIUM

An EDTA preserved whole blood sample was used for white blood cell count by a Coulter Counter Model T 890. Serum and ascitic fluid amylase and lipase were measured by photometry and enzymatic reaction turbidimetry, respectively. ${ }^{20}$ Serum glucose and calcium were measured by an enzymatic GOD (glucose oxygenation-dehydrogenation) method and photometry, respectively. ${ }^{21}$

SERUM TNF- $\alpha$ AND IL- 8

Serum concentrations of TNF- $\alpha$ were measured by using a specific enzyme linked immunosorbent assay (ELISA). A modified version of the Genzyme mouse TNF- $\alpha$ ELISA kit (code no. 80-3807-00) was used..$^{19}$ Serum IL-8 measurements were carried out by a special IL-8 ELISA kit. The method has been published in detail elsewhere. ${ }^{19}$

HISTOLOGICAL EXAMINATION

Paraffin wax embedded sections of the pancreas, right lung, and right kidney were stained with haematoxylin and eosin (H\&E). Sections of lung and kidney were stained for fibrin (picro Mallory), ${ }^{22}$ and lung sections were also stained for elastic tissue (Orcein van Gieson). All sections were evaluated blindly. The specimens were scored for necrosis, inflammatory cellular infiltration, and the presence of vascular thrombosis.

Pancreas

The volume fraction of necrotic pancreatic parenchyma was determined by point counting and expressed as (necrotic tissue/total pancreatic parenchyma $\times 100)$. The part of the pancreas (one third to one half) which was fixed in formalin was divided longitudinally and both pieces were embedded in paraffin wax. Counting was performed on the entire area of an H\&E stained section from each piece using an Olympus microscope connected to a screen by a video camera. Objective magnification was $\times 4$ and each field of vision contained 36 points.

The inflammatory response to necrosis was evaluated semiquantitatively as follows: grade 0 = absent; grade 1 = slight focal inflammation;

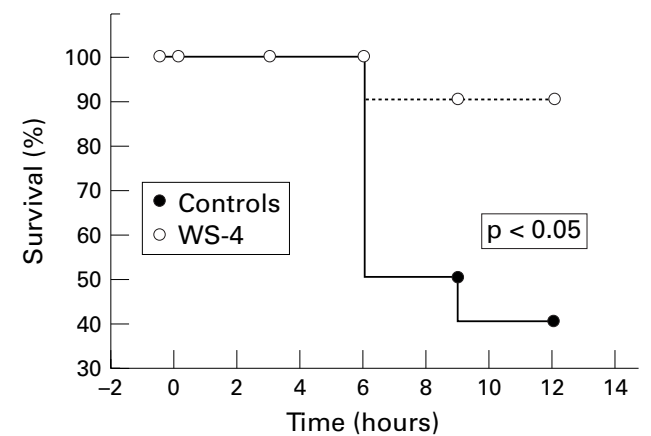

Figure 1 Survival curves for the 12 hour observation period. Fifty per cent of the untreated rabbits were alive at $9 \mathrm{~h}$ and only $40 \%$ at $12 \mathrm{~h}$, whereas $90 \%$ of WS-4 treated rabbits were alive at $12 \mathrm{~h}(p<0.05)$. 

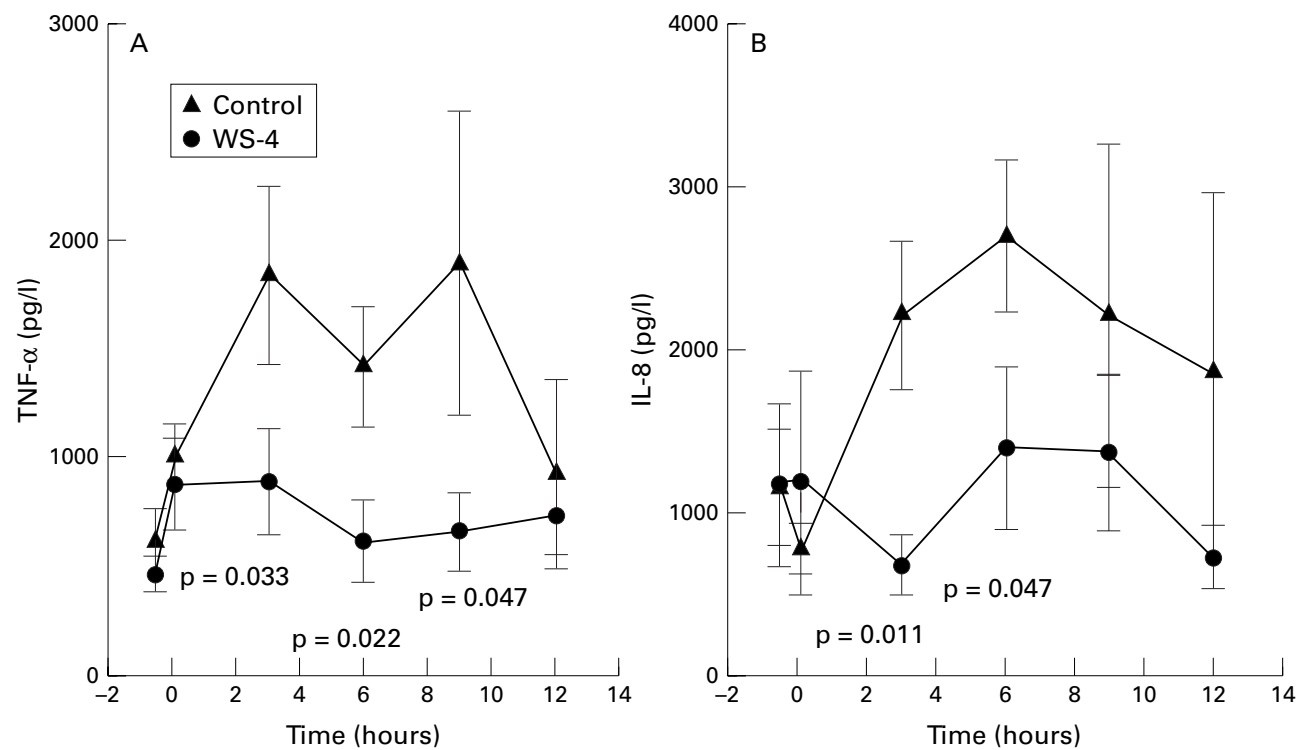

Figure 2 (A) Serum TNF-a concentrations showing a biphasic curve in controls. with peaks at 3 and 9 h. Pretreatment with WS-4 resulted in a significant reduction in TNF-a concentrations from 3 to $9 h$. (B) Serum concentratons of IL-8 increased steadily from 3 to 6 h in controls but a significant reduction was observed in the WS-4 treated group.

grade $2=$ moderate granulocyte margination of necrosis; grade $3=$ widespread and pronounced granulocytic margination of necrosis. The amount of fat necrosis was also semiquantitated: grade $0=$ absent; grade $1=$ less than one third of peripancreatic fat showing necrosis; grade 2 from one to two thirds of fat with necrosis; grade $3=$ more than two thirds of peripancreatic fat is necrotic. Finally, the presence of vascular thrombosis was also described.

Lungs

In $\mathrm{H} \& \mathrm{E}$ stained lung sections from each animal, the degree of oedema was semiquantitatively graded: grade $0=$ none; grade $1=$ slight oedema of the alveolar walls; grade $2=$ moderate oedematous thickening of alveolar walls with occasional alveoli containing coagulated oedema fluid; grade 3 = extensive occurrence of alveolar and interstitial oedema. The number of neutrophils (PMNs) per high power field $(\times 750)$ was counted in 10 random fields of alveolar lung tissue, avoiding fields containing bronchi or large vessels. The presence of margination of PMNs in vasular lumina was also noted. In some of the animals (sham operated ${ }^{19}$ as well as animals with experimental acute pancreatitis) focal interstitial mononuclear infiltrates were found in the lungs. These changes were ascribed to an asymptomatic infection with Bordetella bronchiseptica which can regularly be cultured from the airways of

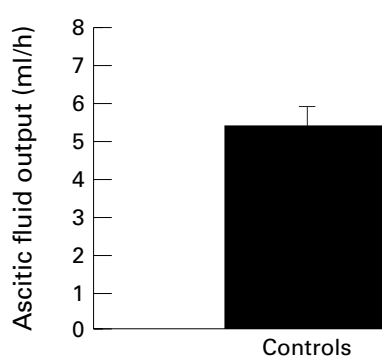

$p=0.002$

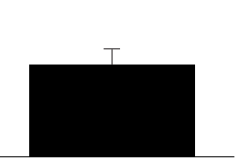

Figure 3 Output of ascitic fluid. laboratory rabbits, and is known to cause such infiltrates. ${ }^{23}$ The infiltrates were semiquantitat as follows: $0=$ none; $1=$ few; $2=$ moderate; 3 $=$ pronounced. In sections stained for fibrin, the presence or absence of capillary thrombosis or thrombosis of larger vessels was noted.

\section{Kidney}

In sections stained for fibrin, the presence or absence of glomerular microthrombi was recorded.

IMMUNOHISTOCHEMICAL DETECTION OF $\mathrm{CD} 11 \mathrm{~b} / \mathrm{CD} 18$

Biopsy specimens from the lung and pancreas were snap frozen in liquid nitrogen and kept at $-80^{\circ} \mathrm{C}$ until processing. The procedure has been described in detail elsewhere. ${ }^{1924}$ The
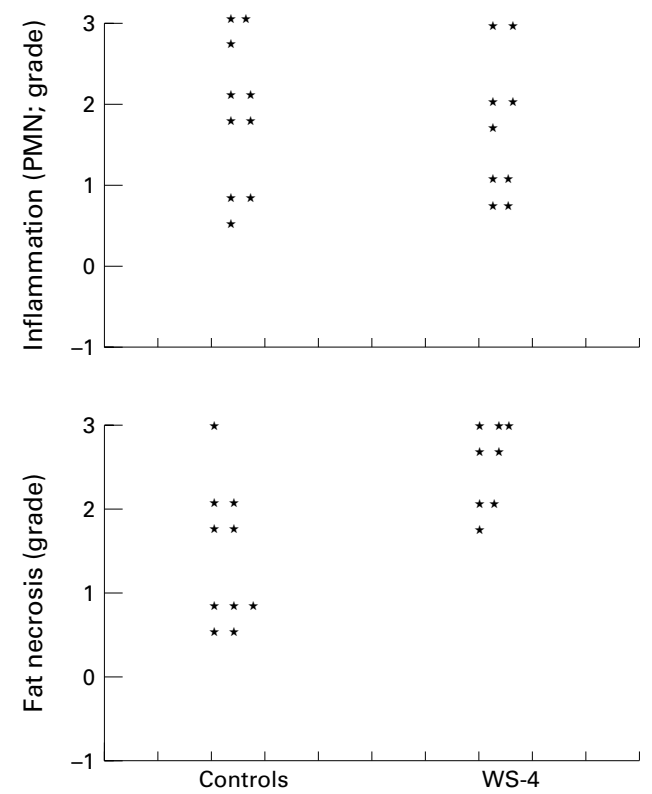

Figure 4 Semiquantitation of the granulocytic inflammatory response to necrosis in pancreatic tissue. 

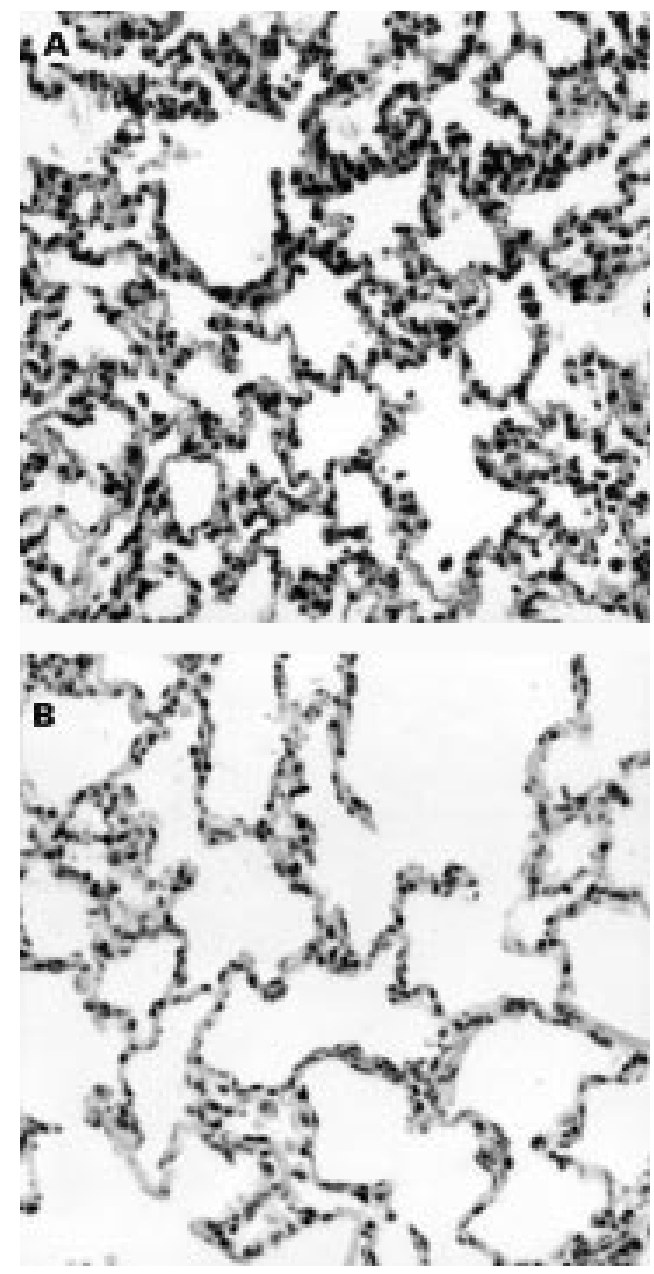

Figure 5 (A) Alveolar lung tissue from a control animal with grade 2 oedema and pronounced infiltration of the alveolar walls with inflammatory cells, which at high magnification are identified as mainly granulocytes. (B) Alveolar lung tissue from a WS-4 treated animal. There is grade 1 oedema and a less intense infiltration of the alveolar walls. Haematoxylin and eosin; original magnification $\times 190$.

immunohistochemical staining of the lung tissue was quantified by computer using random selection by a grid. Due to the severe destuction of the pancreas, counting for statistical purposes was not possible in this organ.

STATISTICAL ANALYSIS

Results are expressed as mean (SEM). Differences between the two groups were determined using the non-parameteric Mann-Whitney rank sum test and/or the unpaired Student's $t$ test. A p value less than 0.05 was considered significant.

\section{Results}

SURVIVAL

Only one rabbit $(10 \%)$ in the WS- 4 treated group died before reaching the end of the 12 hour observation period. In the control group, however, six rabbits $(60 \%)$ died between six and nine hours after induction of acute pancreatitis $(\mathrm{p}<0.05$; fig 1$)$.

SERUM AMYLASE AND LIPASE

In all animals, serum amylase and lipase manifested a steady significant increase with time;
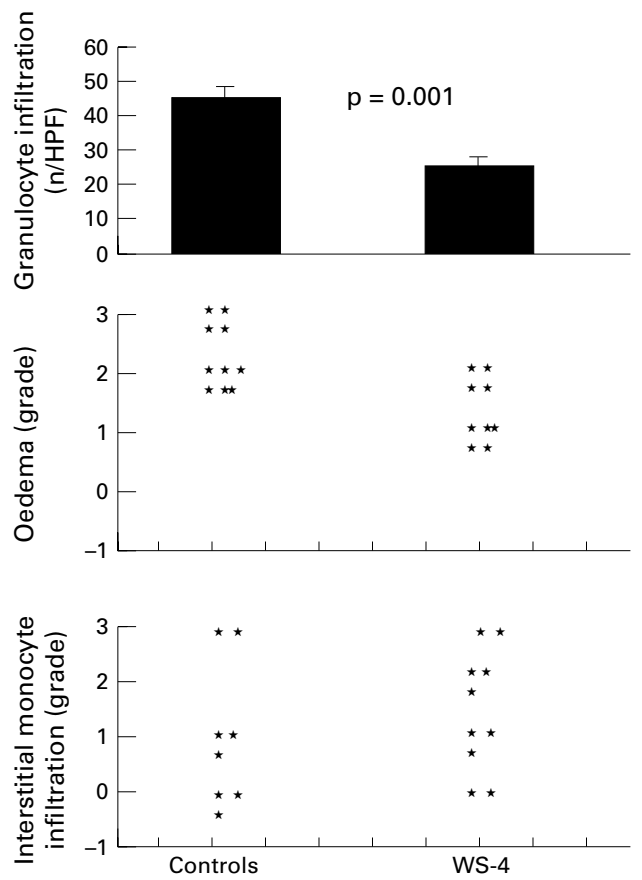

Figure 6 Number of granulocytes per high power field of lung sections (10 random fields), and semiquantitative evaluation of oedema and interstitial monocyte infiltration.

peak concentrations were reached six hours after the induction of acute pancreatitis (amylase from 740 (65) to 16200 (3800) U/1, $\mathrm{p}<0.001$; lipase from 820 (54) to 12300 (3600) U/1, p<0.001). WS-4 pretreatment did not significantly alter the concentration of pancreatic enzymes in plasma throughout the whole observation period.

SERUM CALCIUM AND GLUCOSE

Serum calcium concentrations decreased with time in both groups of animals with no significant difference between the groups (controls: from $3.3(0.2)$ at time 0 to $2.4(0.3) \mathrm{mmol} / \mathrm{l}$ at 12 hours; WS-4 treated animals: from $3.3(0.2)$ at time 0 to $2.7(0.1) \mathrm{mmol} / \mathrm{l}$ at 12 hours). Control animals exhibited hyperglycaemia with a maximum level six hours after the induction of acute pancreatitis, with a subsequent gradual decline to the basal level after 12 hours. WS-4 treated rabbits showed notable hypoglycaemia; the difference compared with control animals was significant after six hours (15.6 (3.7) versus $6.1(0.2) \mathrm{mmol} / \mathrm{l} ; \mathrm{p}=0.011)$.

LEUCOCYTE COUNT AND SERUM TNF- $\alpha$ AND IL- 8 Control animals were significantly leucopenic three hours after the induction of acute pancreatitis (from $5.8(0.3) \times 10^{9} / 1$ to $2.2(0.3)$ $\left.\times 10^{9} / 1\right)$; the leucocyte count increased with time but never reached the basal count. In WS- 4 treated animals, the leucocyte count was not affected; the difference was not significant compared with controls.

Injection of $5 \%$ bile acid into the pancreatic duct and duct ligation resulted in a dramatic elevation in both TNF- $\alpha$ and IL-8 (fig 2) concentrations in the blood. TNF- $\alpha$ serum concentration showed a biphasic curve with maximum concentrations reached after three and 

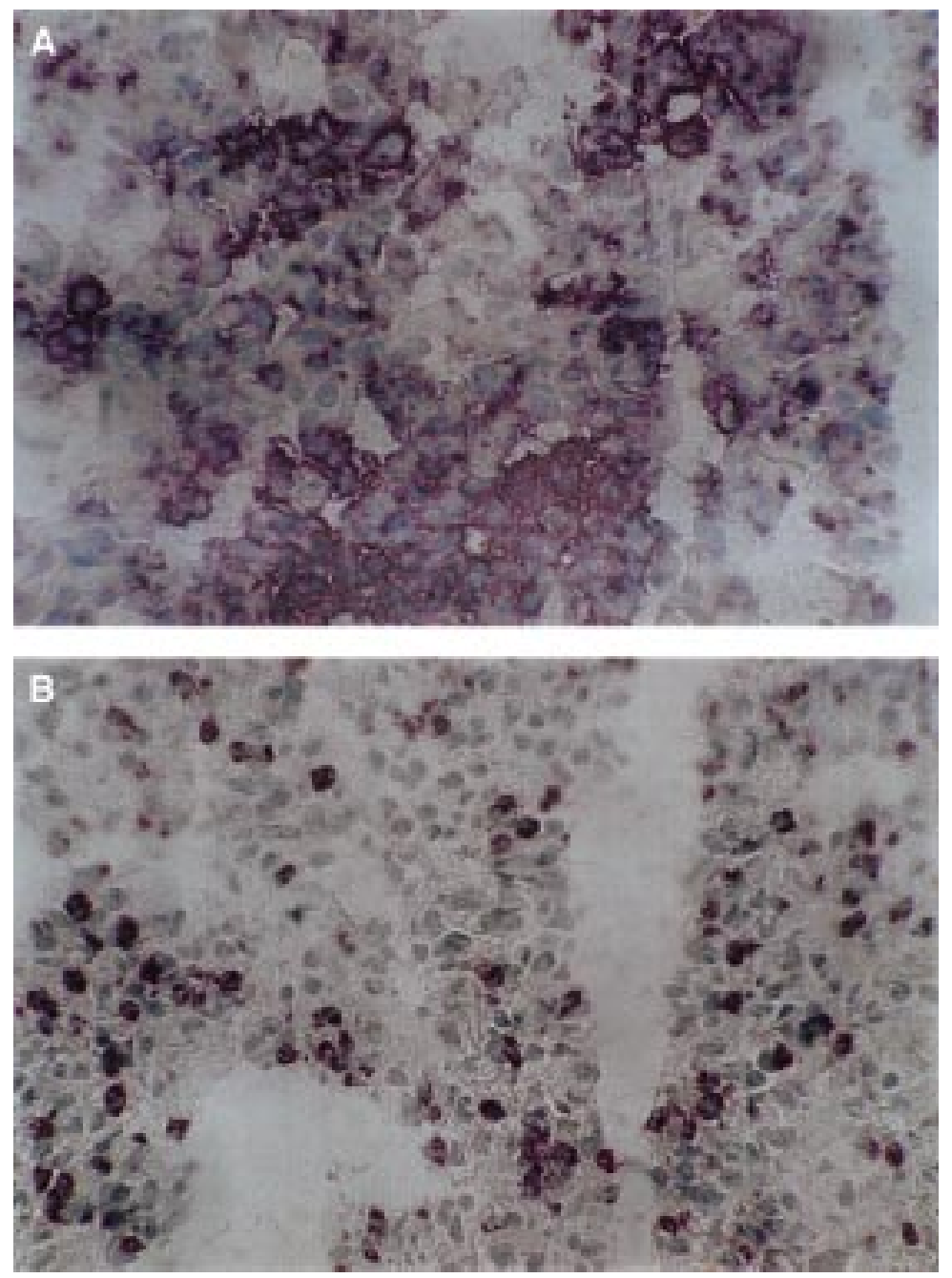

Figure 7 Distribution of CD18 expressing leucocytes in lungs of controls (A) and WS-4 treated animals (B). Streptavidin alkaline phosphatase technique, positive staining appears as red, counterstained with haematoxylin. Bar, $10 \mu \mathrm{m}$.

nine hours, while IL-8 showed a steady increase from three to six hours after the induction of acute pancreatitis. Pretreatment of rabbits with WS-4 significantly reduced the serum concentrations of $\mathrm{TNF}-\alpha$ from three to nine hours $(\mathrm{p}=0.033, \mathrm{p}=0.022$, and $\mathrm{p}=0.047$, respectively) and reduced IL-8 serum concentrations at three and six hours $(p=0.011$ and $\mathrm{p}=0.047$, respectively).

ASCITIC FLUID

Pretreatment of animals with WS-4 resulted in a significant reduction in ascitic fluid output (fig 3). Significant changes were also observed in the concentrations of amylase and lipase in ascitic fluid (data not shown).

\section{MORPHOLOGICAL FINDINGS}

Pancreas

Both groups of animals showed the same degree of widespread parenchyma coagulative necrosis in the pancreas. In the control group, the mean volume of necrotic tissue was 73.5 (2.9)\%. In the WS-4 group, the mean value was $66.8(5.1) \%$. There was also no difference between the two groups with respect to the inflammatory response in the pancreas (fig 4). The individual differences found in the degree of inflammation seemed to be related to the time of survival after bile acid injection. Thus, control animals surviving for only six or nine hours showed practically no inflammation (grade 0 or 1 ), whereas in most animals with longer survival in both groups, a grade 2 or 3 inflammatory response was seen. However, only 4/9 animals in the WS- 4 group which survived for 12 hours showed grade 1 inflammation. The same was true for the degree of fat necrosis (fig 4). Thrombosis of pancreatic vessels was found in 6/10 control animals and 7/9 WS-4 treated animals (NS).

\section{Lungs}

In the lungs of control animals (fig 5), a moderate to severe oedema was observed as well as a pronounced infiltrate of neutrophils (PMNs) in the alveolar walls. These changes were reduced significantly in the WS-4 treated group (figs 5 and 6). The PMNs were scattered diffusely throughout the lung tissue and not particularly related to the interstitial mononuclear infiltrates found in most animals (fig 6). In most control animals (9/10) and WS-4 treated animals $(8 / 9)$, a more or less pronounced leucocytic vascular margination and perivascular infiltrates of PMNs were noted. Foci of vascular thrombosis in large vessels and/or microthrombi were seen in the control group (6/10) and the WS-4 group (5/9).

\section{Kidneys}

In the control group, six animals showed few glomerular microthrombi, while animals in the WS-4 treated group did not show signs of vascular thrombosis. In other respects, the kidneys appeared normal.

\section{IMMUNOHISTOCHEMICAL DETECTION OF}

\section{$\mathrm{CD} 1 \mathrm{~b} / \mathrm{CD} 18$}

The immunohistochemical presence of CD11b and CD18 was noted in all animals examined.

In the lung, CD11b was detected in all control animals. It gave rise to a cytoplasmic staining localised to cells which were mainly neutrophils. CD11b positive cells were observed throughout the lung tissue. In WS-4 treated animals, CD11b positive cells were observed in the lung, but were greatly reduced in number compared with controls $(\mathrm{p}<0.001$; table 1). They were mainly localised around bronchioles and not in the lung tissue. CD18 was detected in all animals in both lung and pancreatic tissues. Although more cells expressed CD18 than CD11b, serial sections revealed that cells seemed to be both CD11b/ CD18 positive, suggesting that this complex is of importance for the inflammatory process in rabbits. The highest expression of CD18 was observed in the lung (fig 7) and pancreas of control animals. The WS- 4 treated animals (fig 7) had a greatly reduced number of CD18 positive cells in lung sections compared with controls $(p<0.001$; table 1$)$.

The pancreas was too severely destroyed to allow counting for statistical purposes, but CD1 $1 \mathrm{~b}$ and CD18 positive cells were observed in glands of all control animals and some WS-4 treated animals. 
Table 1 Number of CD11b and CD18 positive cells in rabbit lung tissue (cell number in computerised units)

\begin{tabular}{lll}
\hline & $C D 11 b$ & $C D 18$ \\
\hline Controls & $7.3(2.7)$ & $42.5(8.0)$ \\
WS-4 treated animals & $4.0(1.1)$ & $8.0(3.0)$ \\
p Value & $<0.001$ & $<0.001$ \\
\hline
\end{tabular}

\section{Discussion}

The present study showed that WS-4 injection prior to the induction of severe acute pancreatitis in rabbits caused the following: (1) a significant reduction in the serum concentration of the proinflammatory cytokines $\mathrm{TNF}-\alpha$ and IL-8; (2) notable down regulation of the adhesion molecule complex CD11b/CD18 in lung tissue; (3) significant attenuation of the inflammatory response in lung tissue as evidenced by a reduction in neutrophil infiltration and vascular thrombosis as well as oedema formation in the lungs; (4) a significant reduction in ascitic fluid output and its content of amylase and lipase; (5) a significant increase in animal survival for the 12 hour observation period; (6) no effect on the percentage of pancreatic parenchymal necrosis or the amount of neutrophil infiltration in the pancreas; and (7) an insignificant effect on the peripheral leucocyte count as well as on the other biochemical parameters of acute pancreatitis (serum amylase, lipase, and calcium).

These findings suggest that IL-8, which has been shown to be produced locally by pancreatic acinar and ductal cells, ${ }^{19}$ might be primarily responsible for the notable infiltration of neutrophils into the pancreas and lung tissue, leading to development of acute lung injury (ALI) and production of a massive amount of ascitic fluid in rabbits infused with $5 \%$ bile acid.

The importance of proinflammatory cytokines in the pathogenesis of acute pancreatitis and its systemic complications is becoming increasingly apparent. These cytokines would be expected to act locally to aggravate the pancreatitis process, and both locally and systemically to increase capillary permeability and promote leucocyte adherence and extravasation. ${ }^{52}$ Recently, IL-8 has been shown to be elevated in the serum of patients with complicated acute pancreatitis. ${ }^{14}{ }^{15}$ High serum IL-8 values can be detected early in the course of severe disease, several hours before the rise in polymorphonuclear elastase. ${ }^{11}$ In one study, serum IL-8 displayed the earliest peak of any of the currently available inflammatory markers in acute pancreatitis. ${ }^{14} \mathrm{~A}$ potential role for IL-8 in the pathogenesis of severe acute pancreatitis has therefore been suggested. Only one previous experimental study has focused on the role of IL-8 in acute pancreatitis by measuring its systemic blood concentration in rats with caerulein induced or closed duodenal loop pancreatitis. ${ }^{13}$ The aggravation of pancreatitis in adrenalectomised rats was accompanied by high concentrations of serum IL-8. Administration of exogenous hydrocortisone suppressed the elevation of serum IL-8 concentrations and decreased both the severity of pancreatitis and mortality of rats in these models. ${ }^{13}$ Although the actual role of IL-8 in the inflammatory process remains to be defined, the secretion of IL- 8 by a wide variety of cells and the range of its biological activities, suggest that IL-8 can play an important role in the progression of acute pancreatitis. IL- 8 has been reported to induce the full pattern of responses in chemotactically stimulated neutrophils (activation of the motile apparatus and directional migration, ${ }^{6}$ expression of surface adhesion molecules, ${ }^{26}$ release of proteolytic enzymes, and production of reactive oxygen metabolites ${ }^{27}$ ). Collectively, these effects of IL-8 might be responsible for its active role in the propagation of acute pancreatitis from a localised inflammatory necrosis into a whole body inflammation.

The role of neutrophils in inflammation has been extensively studied. It has been reported that polymorphonuclear leucocytes are essential for the vascular permeability response to chemotactic substances. ${ }^{28}$ Neutrophils have also been shown to play an important role in tissue destructive events in different inflammatory conditions through the release of their proteolytic enzymes such as PMN elastase and the production of lethal oxygen metabolites. ${ }^{29}$ IL-8 recruited and activated neutrophils have been reported to play a crucial role in the development of adult respiratory distress syndrome (ARDS) in at risk patients, particularly those with severe sepsis or severe necrotising acute pancreatitis. ${ }^{10}$ In another study, ARDS patients with the highest concentrations of IL-8 had a higher mortality, indicating that IL-8 contributes to a poor outcome directly through recruitment and activation of neutrophils in the lung. ${ }^{30}$ Furthermore, an influx of leucocytes into the inflamed pancreas, monitored by leucocyte scintigraphy, has been shown in patients with severe acute pancreatitis. ${ }^{31}$ Therefore, the high mortality rate associated with fatal haemorrhagic pancreatitis can also be explained by activation of leucocytes and their immigration into the pancreas, thus aggravating the local process of pancreatitis and initiating the development of systemic complications of acute pancreatitis. ${ }^{3}$ In acute inflammation, neutrophils migrate down a concentration of chemotactic factors. ${ }^{29}$

IL-8 possesses a potent chemotactic activity compared with other monokines. ${ }^{32}$ Administration of WS-4 in a model of lung reperfusion injury in rabbits prevented neutrophil infiltration and tissue injury. ${ }^{18}$ Similarly, application of an antibody to human IL-8 blocked recruitment of neutrophils and protected against lung injury initiated by E-selectin dependent recruitment of neutrophils in rats. ${ }^{33}$ Therefore, our findings, together with the above mentioned observations, strongly support the role of IL-8 in the development of ALI complicating severe acute pancreatitis in our rabbit model, through the recruitment and activation of neutrophils. Clinically, administration of lexipafant, a platelet activating factor antagonist, has reduced organ dysfunction and suppressed serum concentrations of IL-8 in patients with severe acute pancreatitis. ${ }^{34}$ 
IL-8 is also a potent activator of the adhesion complex CD11b/CD18 which has been shown to play a crucial role in neutrophil adhesivness and transendothelial neutrophil extravasation. ${ }^{26}$ A pivotal role for the adhesion complex CD11b/ CD18 has been shown in neutrophil induced tissue damage-for example, in reperfusion injury and endotoxic shock. ${ }^{35}{ }^{36} \mathrm{Up}$ regulation of the adhesion complex CD11b/CD18 in the lungs of animals with $5 \%$ bile acid induced acute pancreatitis represents another possible mechanism by which IL- 8 may cause progression of acute pancreatitis in rabbits. Blocking the effects of CD18 in a rat model of bile acid induced acute pancreatitis prevented the development of ALI and improved survival. ${ }^{37}$ Similarly, administration of LFA-1 (CD11a/CD18) antibody attenuated superoxide anion release from polymorphonuclear leucocytes and thus reduced the severity of ALI in rats with bile acid induced pancreatitis. ${ }^{38}$ Significant down regulation of the adhesion complex CD11b/CD18 in lung tissue by WS- 4 pretreatment further emphasises the potential role of IL-8 in perpetuating the severity of acute pancreatitis.

The role of TNF- $\alpha$ in the pathogenesis of severe acute pancreatitis has been shown in several experimental studies, mainly in rats and mice. ${ }^{39} 40$ Serum and intrapancreatic concentrations of TNF- $\alpha$ have been shown to be elevated in mice with caerulein induced acute pancreatitis. $^{39}$ Furthermore, TNF- $\alpha$ mRNA has been shown to be induced in distant organs during the process of bile acid induced acute pancreatitis in rats. ${ }^{41}$ Serum TNF- $\alpha$ is not however constantly increased in complicated cases of human acute pancreatitis. ${ }^{42}$ TNF- $\alpha$ is a pleiotropic cytokine and many of its actions have been suggested as possible mechanisms for the systemic cellular events that occur during acute pancreatitis. These include: cytotoxic effects on endothelial cells ${ }^{43}$; increased adherence of neutrophils to endothelial cells ${ }^{44}$; increased vascular permeability; and induction of acute lung injury in a neutrophil dependent manner. ${ }^{45}$

Although TNF- $\alpha$ is a potent inducer of IL-8, particularly from endothelial cells, ${ }^{46} \mathrm{TNF}-\alpha$ induced transendothelial neutrophil migration is IL- 8 dependent. ${ }^{47}$ Compared with TNF- $\alpha$, IL-8 has strong chemotactic and stimulating activity particularly on neutrophils. ${ }^{32}$ In our model, administration of the monoclonal antiIL-8 antibody resulted in a significant reduction in the serum concentration of TNF- $\alpha$, which is most likely to be due to the inhibitory effect of the antibody on the inflammatory process in general. This might explain the attenuation of ALI and the improved survival in animals pretreated with WS-4.

Administration of the monoclonal anti-IL-8 antibody (WS-4) did not however significantly affect the local process of pancreatitis in rabbits. Histologically, both treated and untreated rabbits showed equivalent amounts of pancreatic necrosis and neutrophil infiltration. Biochemically, the blood concentrations of amylase, lipase, and calcium remained unchanged in both experimental animal groups. Considering the technique of induction of acute pancreatitis, the design of the experiment, and the detergent effect of the bile acid injected, these findings were not unexpected. It is also important to note that bile salts are very potent stimuli for oxygen free radical production and that detergents and crystals in bile would be extremely powerful chemotaxins. ${ }^{48}$ These effects of bile, together with the presence of other active chemokines in the circulation not affected by WS-4, might also explain the poor effect of WS-4 pretreatment on local pancreatic inflammation. However, in a similar model of bile induced acute pancreatitis in rats, blocking the effects of TNF- $\alpha$ by a polyclonal anti-TNF- $\alpha$ antibody in one study, ${ }^{49}$ and IL-1 by IL-1 receptor antagonist in another, ${ }^{50}$ resulted in an amelioration of the physiological and biochemical changes of acute pancreatitis with an overall improvement in animal survival, but without significant effects on local pancreatic damage..$^{49}$

Our findings, together with those of the above mentioned studies, ${ }^{48} 49$ are consistent with the hypothesis that the predominantly macrophage derived inflammatory cytokines, including IL-8, play a critical role in the progression of acute pancreatitis and the development of its associated systemic complications in a bile induced model of acute pancreatitis in the rabbit.

The authors wish to express their deepest gratitude to Walter Gyldenløwe and his staff at the research farm, Aarhus University, for their support and unfailing interest. The study was supported by grants from NYCOMED-DAK, Velux Foundation, Boels Foundation, and Auto Suture, Norden. Bent Deleuran is supported by grants from the Danish Rheumatism Association and the Mads Clausens Foundation.

1 Steinberg W, Tenner S. Acute pancreatitis. N Engl f Med 1994;330:1198-210.

2 Renner JG, Savage WT, Pantoja JL, et al. Death due to acute pancreatitis: a retrospective analysis of 405 autopsy cases. Dig Dis Sci 1985;30:1005-18.

3 Rinderknecht $\mathrm{H}$. Fatal pancreatitis, a consequence of excessive leucocyte stimulation? Int f Pancreatol 1988;3:105-12.

4 Kusske AM, Rongione AJ, Reber HA. Cytokines and acute pancreatitis. Gastroenetrology 1996;110:639-42.

5 Gross V, Lesser H-G, Heinisch A, et al. Inflammatory mediators and cytokines-new aspects of the pathophysiology and assessment of severity of acute pancreatitis? Hepatogastroenterology 1993;40:522-30.

6 Baggiolini M, Walz A, Kunkel SL. Neutrophil-activating peptide-1/interleukin-8, a novel cytokine that activates neutrophils. F Clin Invest 1989;84:1045-9.

7 Larsen CG, Anderson AO, Appella E, et al. The neutrophilactivating protein (NAP-1) is also chemotactic for $\mathrm{T}$ ymphocytes. Science 1989;243:1464-6.

8 Endo H, Akahoshi T, Takagishi K, et al. Elevation of interleukin-8 (IL-8) levels in joint fluids of patients with rheumatoid arthritis and the induction by IL-8 of leucocyte infiltration and synovitis in rabbit joints. Lymphokine Cytokine Res 1991;10:245-52.

9 Hack CE, Hart M, Strack van Schijndel RJM, et al. Interleukin-8 in sepsis: relation to shock and inflammatory mediators. Infect Immun 1992;60:2835-42.

10 Donnelly SC, Strieter RM, Kunkel SL, et al. Interleukin-8 and development of adult respiratory distress syndrome in at-risk patient groups. Lancet 1993;341:643-7.

11 Gross V, Andreesen R, Leser H-G, et al. Interleukin-8 and neutrophil activation in acute pancreatitis. Eur f Clin Invest 1992;22:200-3

12 Ventrucci $M$, Gionchetti P, Baraldini M, et al. Behaviour of serum interleukin-8 during the course of acute pancreatitis: comparison with serum interleukin-6 and C-reactive protein [abstract]. Digestion 1993;54:314.

13 Abe R, Shimosegawa T, Kimura K, et al. The role of endogenous glucocorticoids in rat experimental models of acute pancreatitis. Gastroenetrology 1995;109:933-43.

14 Kingsnorth AN, Galloway SW, Formela LJ. Randomized, double-blind phase II trial of Lexipafant, a plateletactivating factor antagonist, in human acute pancreatitis. Br f Surg 1995;82:1414-20.

15 McKay CJ, Gallagher G, Brooks B, et al. Increased monocyte cytokine production in association with systemic complications in acute pancreatitis. Br F Surg 1996;83:919-23. 
16 Ko Y-C, Mukaida N, Panyutich A, et al. A sensitive enzymelinked immunosorbent assay for human interleukin-8. $f$ linked immunosorbent assay for han

17 Larsen CG, Thomsen MK, Gesser B, et al. The delayedtype hypersensitivity reaction is dependent on IL-8: inhibition of a tuberculin skin reaction by an anti-IL-8 monoclonal antibody. F Immunol 1995;155:2151-7.

18 Sekido N, Mukaida N, Harada A, et al. Prevention of lung reperfusion injury in rabbits by a monoclonal antibody against interleukin-8. Nature 1993;365:654-7.

19 Osman MO, Lausten SB, Jacobsen NO, et al. Graded experimental acute pancreatitis: monitoring of a renewed rabbit model focusing on the production of TNF- $\alpha$, interleukin-8 and CD11b/CD18. Eur F Gastroenterol Hepatol 1998; in press.

20 Ziegehorn J, Neumann U, Knitsch KW, et al. Determination of serum lipase. Clin Chem 1979;25:1067.

21 Gindler EM, King JD. Rapid colorimetric determination of calcium in biologic fluids with methylthymol blue. $A m$ f Clin Pathol 1972;58:376-82.

22 Carstairs KC. The identification of platelets and platelet antigen in histological sections. F Pathol Bacteriol 1965;90: antigen in

23 Uzal FA, Feinstein RE, Rehbinder C, et al. A study of lung lesions in asymptomatic rabbits naturally infected with B. bronchiseptica. Scandinavian fournal of Laboratory Animal Science 1989;16:3-13.

24 Deleuran B, Lemche P, Kristensen M, et al. Localization of interleukin- 8 in the synovial membrane, cartilage-pannus junction and chondrocytes in rheumatoid arthritis. Scand $\mathcal{f}$ Rheumatol 1994;23:2-7.

25 Scholmerich J. Interleukins in acute pancreatitis. Scand $\mathcal{F}$ Gastroenterol 1996;31 (suppl 219):37-42.

26 Detmers PA, Lo SK, Olsen Egbert E, et al. Neutrophilactivating protein-1/interleukin-8 stimulates the binding activity of the leucocyte adhesion receptor CD11b/CD18 on human neutrophils. 7 Exp Med 1990;171:1155-62.

27 Djeu JY, Matsushima K, Oppenheim JJ, et al. Functional activation of human neutrophils by recombinant activation of human neutrophils by recombinant Immunol 1990;144:2205-10.

28 Wedmore CV, Williams TJ. Control of vascular permeability by polymorphonuclear leucocytes in inflammation. Nature 1981;289:646-50.

29 Weiss SJ. Tissue destruction by neutrophils. $N$ Engl f Med 1989;320:365-76.

30 Miller EJ, Cohen AB, Nagao S, et al. Elevated levels of NAP-1/interleukin-8 are present in the air spaces of patients with adult respiratory distress syndrome and are associated with increased mortality. Am Rev Respir Dis 1992;146:427-32.

31 Scholmerich J, Schumichen C, Lausen M, et al. Scintigraphic assessment of leucocyte infiltration in acute pancreatitis using technetium-99m-hexamethyl propylene amine oxine as leucocyte label. Dig Dis Sci 1991;36:65-70.

32 Willem J, Joniau M, Cinque S, et al. Human granulocyte chemotactic peptide (IL-8) as a specific neutrophil chemotactic peptide (IL-8) as a specific neutrophil degranulator: comp

33 Mulligan MS, Jones ML, Bolanowski MA, et al. Inhibition of lung inflammatory reactions in rats by an anti-human IL-8 antibody. F Immunol 1993;150:5585-95.
34 Kingsnorth AN, Galloway SW, Formela LJ. Randomized, double-blind phase II trial of Lexipafant, platelet-activating factor antagonist, in human acute pancreatitis. Br f Surg 1995;82:1414-20.

35 Jaeschke T, Farhood A, Smith W. Neutrophil-induced liver cell injury in endotoxic shock is a CD11b/CD18dependent mechanism. Am f Physiol 1991;261:G1051-6.

36 Veder NB, Winn RK, Rice CL, et al. A monoclonal antibody to the adherence-promoting leucocyte glycoprotein, $\mathrm{CD} 18$, reduces organ injury and improves survival from haemorrhagic shock and resuscitation in rabbits. $\mathcal{F}$ Clin Invest 1988;81:939-44.

37 Inoue S, Nakao A, Kishimoto W, et al. Anti-neutrophil antibody attenuates the severity of acute lung injury in rats with experimental acute pancreatitis. Arch Surg 1995;130:93-8.

38 Inoue S, Nakao A, Kishimoto W, et al. LFA-1 (CD11a/ CD18) and ICAM-1 (CD54) antibodies attenuate superoxide anion release from polymorphonuclear leucocytes in rats with experimental acute pancreatitis. Pancreas 1996;12: 183-8.

39 Grewal HP, Kotb M, El-Din AM, et al. Induction of tumor necrosis factor in severe acute pancreatitis and its subsequent reduction after hepatic passage. Surgery 1994; 115:213-21.

40 Norman JG, Fink GW, Franz MG. Acute pancreatitis induces intrapancreatic tumor necrosis factor gene expression. Arch Surg 1995;130:966-70.

41 Hughes CB, Henry J, Kotb $M$, et al. Up-regulation of TNF- $\alpha$ in the rat spleen following induction of acute pancreatitis. F Surg Res 1995;59:687-93.

42 Banks RE, Evans SW, Alexander D, et al. Is fatal pancreatitis a consequence of excessive leucocyte stimulation? The role of tumor necrosis factor $\alpha$. Cytokine 1991;3:12-16.

43 Laster SM, Wood JG, Gooding LR. Tumor necrosis factor can induce both apoptic and necrotic forms of cell lysis. $f$ Immunol 1988;141:2629-34.

44 Gamble R, Harlan JM, Klebanoff SJ, et al. Stimulation of the adherence of neutrophils to umbilical vein endothelium by recombinant tumor necrosis factor. Proc Natl Acad Sci USA 1985;82:8667-71.

45 Stephens KE, Ishizaka A, Larrick JW, et al. Tumor necrosis actor causes increased vascular permeability and edema. Am Rev Respir Dis 1988;138:1300-30.

46 Lowry SF. Cytokine mediators of immunity and inflammation. Arch Surg 1993;128:1235-41.

47 Smart SJ, Casale TB. TNF- $\alpha$-induced transendothelial neutrophil migration is IL-8 dependent. Am $\mathcal{f}$ Physiol 1994;266:L238-45.

48 Craven PA, Pfanstiel J, DeRubertis FR. Role of reactive oxygen in bile salt stimulation of colonic epithelial proliferation. $\mathcal{F}$ Clin Invest 1986;77:850-9.

49 Grawal HP, El-Din AM, Gaber L, et al. Amelioration of the physiologic and biochemical changes of acute pancreatitis using an anti-TNF- $\alpha$ polyclonal antibody. Am F Surg 1994; 167:214-19.

50 Tanaka N, Murata A, Uda K-I, et al. Interleukin-1 receptor antagonist modifies the changes in vital organs induced by acute necrotizing pancreatitis in a rat experimental model. Crit Care Med 1995;23:901-8. 